\title{
Hiperplasia hipofisaria secundaria a hipotiroidismo primario
}

\author{
Pituitary hyperplasia secondary to primary hipothyroidism
}

\author{
Oscar Castillo ${ }^{1,4}$, Milena Cárdenas², Lida Tello ${ }^{3}$, Ricardo Florián ${ }^{4}$ \\ ${ }^{1}$ Hospital Nacional Arzobispo Loayza, Lima, Perú. \\ ${ }^{2}$ Residente de Endocrinología, Universidad Nacional Mayor de San Marcos. \\ ${ }^{3}$ Oficina de Epidemiologia y Salud Ambiental, Hospital Nacional Arzobispo Loayza.
}

${ }^{4}$ Clínica Stella Maris, Lima, Perú.

\begin{abstract}
Resumen
Paciente mujer de 15 años referida por diagnóstico de hipotiroidismo e hiperprolactinemia, con tratamiento irregular para ambas patologías, quien presentó pérdida de conocimiento en dos oportunidades, motivo por el cual se le indicó resonancia magnética (RM) de cerebro, en la cual se evidenció imagen tumoral hipofisaria que desplazaba el quiasma óptico. Se le indicó tratamiento con levotiroxina 50 ug por 1 semana, luego 100 ug diario. Los controles hormonales posteriores mostraron normalización, la RM de control evidenció disminución de tamaño de la imagen tumoral en aproximadamente $3 \mathrm{~mm}$, sin repercusión en las estructuras supraselares 0 paraselares, con mejoría de cuadro clínico luego del tratamiento de sustitución con hormona tiroidea.
\end{abstract}

Palabras clave: Hiperplasia hipofisaria, hipotiroidismo, hiperprolactinemia.

Summary

A 15 year-old woman with diagnosis of hypothyroidism and hyperprolactinemia had irregular treatment. She lost conscience twice and magnetic resonance (MR) was indicated. It showed image of pituitary tumor that displaced optic chiasm. She was prescribed levothyroxin $50 \mathrm{ug}$ for one week and then $100 \mathrm{ug}$ daily. Hormonal treatment produced normalization of hormonal values and magnetic resonance evidenced $3 \mathrm{~mm}$ tumor reduction without supraselar extension.

Key words: Pituitary hyperplasia, hypothyroidism, hyperprolactinemia.

\section{An Fac med. 2013;74(1):71-5}

\section{INTRODUCCIÓN}

Existen diversas causas de masas selares y paraselares. El hipotiroidismo primario está relacionado dentro de estas posibilidades con la presencia de 'masas' selares debidas a hiperplasia hipofisaria, en ocasiones con extensión supraselar ${ }^{(1-5)}$. Se producen como resultado de la falta de inhibición hipotálamo hipofisaria por la tiroxina, lo que origina sobreproducción de hormona liberadora de tirotropina (TRH) y consecuentemente incremento de tirotropina (TSH), la cual puede generar hiperplasia hipofisaria. Los adenomas hipofisarios son lesiones intraselares, autónomos, más comunes en adultos. El hipotiroidismo primario no tratado por largo tiempo puede generar injuria hipofisaria irreversible, la cual puede conducir a una deficiencia en una o más hormonas hipofisarias ${ }^{(5)}$. La incidencia de hiperplasia hipofisaria en paciente con hipotiroidismo varía entre 25 y $81 \%$; la alta incidencia (70\%) se da en pacientes con niveles de $\mathrm{TSH} \geq 50 \mathrm{uUI} / \mathrm{mL}^{(5)}$.

A pesar de los recientes avances en técnicas de imagen, utilizando tomografía o resonancia magnética (RM) de hipófisis no es posible distinguir entre los macroadenomas productores de TSH y la hiperplasia de los tirotrofos de la hipófisis. En estos casos, repetir la RM después del tratamiento con levotiroxina puede proporcionar un diagnóstico definitivo y descartar la cirugía, ya que, a diferencia del adenoma hipofisario, la hiperplasia se resuelve con la terapia de reemplazo de hormona tiroidea ${ }^{(3-5)}$. La escasa frecuencia de presentación y el tiempo de seguimiento del presente caso nos motiva a reportarlo.

\section{CASO CLÍNICO}

Paciente mujer de 15 años, natural y procedente de Lima, referida del servicio de medicina interna el día 20/03/95 
por haber presentado dos episodios de pérdida de conocimiento, visión borrosa, cefalea y antecedente de hiperprolactinemia. A los 12 años de edad fue diagnosticada de hipotiroidismo, recibiendo tratamiento con tiroglobulina $65 \mathrm{mg} /$ día (Proloid) por tres meses, y bromocriptina $1,25 \mathrm{mg} /$ día durante un mes, para controlar la hiperprolactinemia. A los 13 años presentó episodio de lipotimia y se diagnosticó hipoglicemia. Nació de parto eutócico, a término, con un peso de $3320 \mathrm{~kg}$, talla $49 \mathrm{~cm}$. Tuvo menarquia a los 11 años, régimen catamenial 5/30, última menstruación en marzo de 1995. La abuela materna sufría de diabetes mellitus tipo 2, madre aparentemente sana, padre con hipertensión arterial y dislipidemia, hermano sano. Al examen físico, la presión arterial era $110 / 60 \mathrm{mmHg}$, pulso 68 por minuto, peso $54,2 \mathrm{~kg}$, talla $1,53 \mathrm{~m}$. La paciente tenía aparente buen estado general, regular estado nutricional y de hidratación. El cuello era central, se palpaba bocio difuso, de consistencia firme, de aproximadamente $60 \mathrm{~g}$, no doloroso. En mamas se evidenció galactorrea bilateral, una gota de secreción serosa. El aparato respiratorio, cardiovascular y abdomen estaban dentro de límites normales. La paciente estaba despierta, lúcida, orientada en tiempo espacio y persona, reflejo aquiliano normal.

La siguiente fue la secuencia de exámenes auxiliares:

- 14/3/95: Prueba de tolerancia a la glucosa basal $92 \mathrm{mg} / \mathrm{dL}$, a los 30' 119 $\mathrm{mg} / \mathrm{dL}$, a los $60^{\prime} 82 \mathrm{mg} / \mathrm{dL}$, a los $90^{\prime}$ $77 \mathrm{mg} / \mathrm{dL}$ y a los $120^{\prime} 70 \mathrm{mg} / \mathrm{dL}$. Hemoglobina $10,2 \mathrm{~g} \%$.

- 25/3/95: Glicemia 87 mg/dL, insulina $10,5 \mathrm{mIU} / \mathrm{mL}$, relación $\mathrm{I} / \mathrm{G}$ 0,12 (normal menor de 0,3).

- 17/5/95: Captación de I-131 a las 2 h $12,9 \%$, a las 24 h $66 \%$, a las $48 \mathrm{~h}$ 65,8\%; captación tiroidea Tc-99m a los 20 minutos $47,5 \%$; gammagrafía de tiroides con Tc-99m, bocio difuso hipercaptador, glándula de 60 a $70 \mathrm{~g}$ (figura 1).

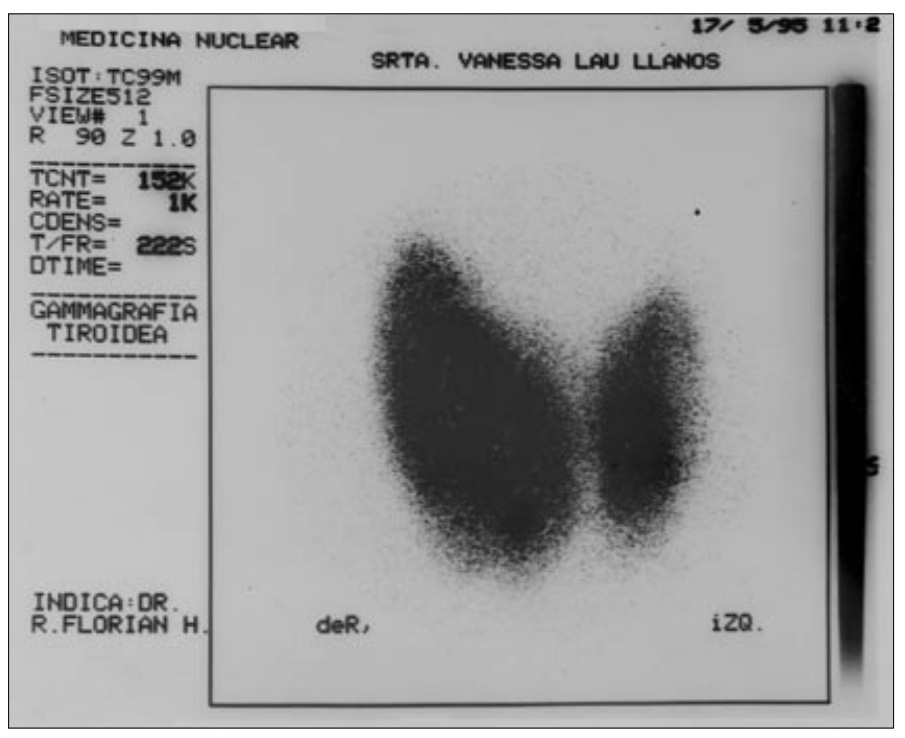

Figura 1. Gammagrafia de tiroides con tecnecio.

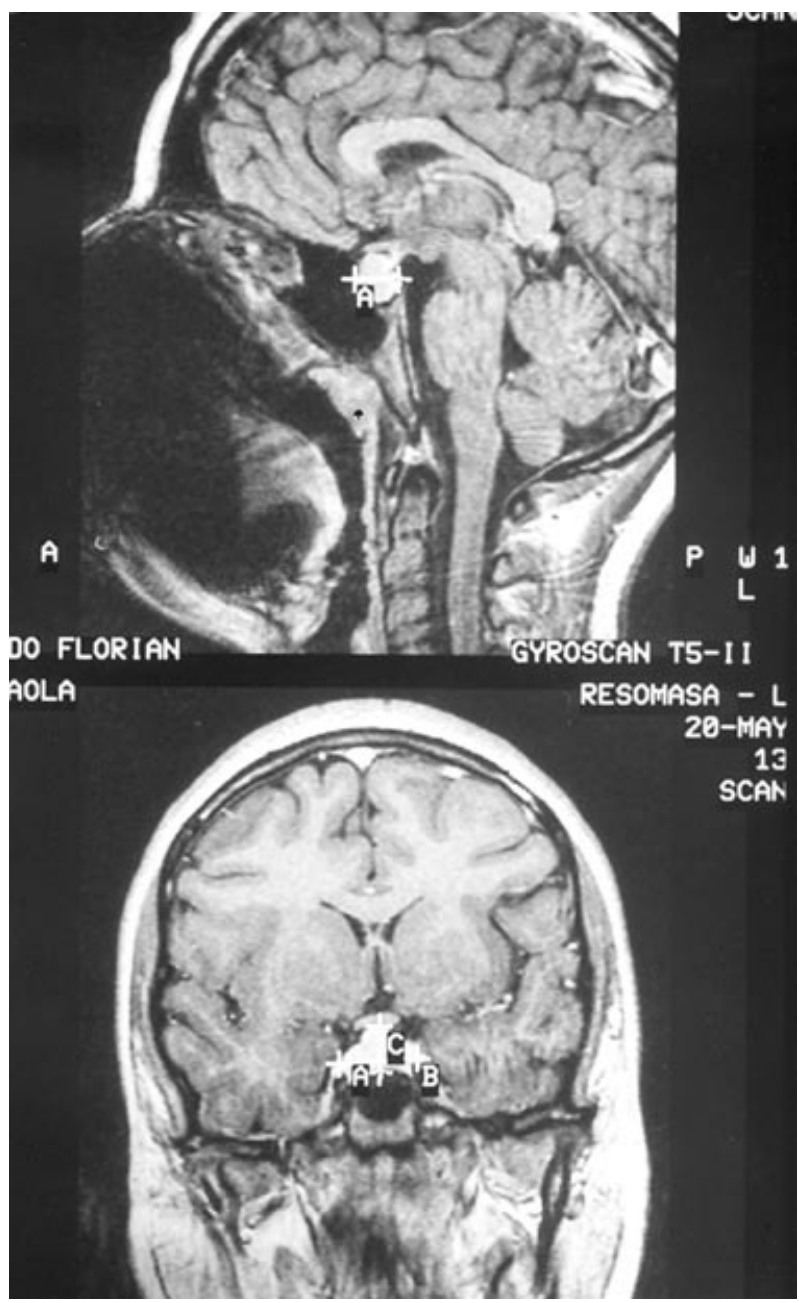

Figura 2. Resonancia magnética cerebral antes del tratamiento con levotiroxina. 


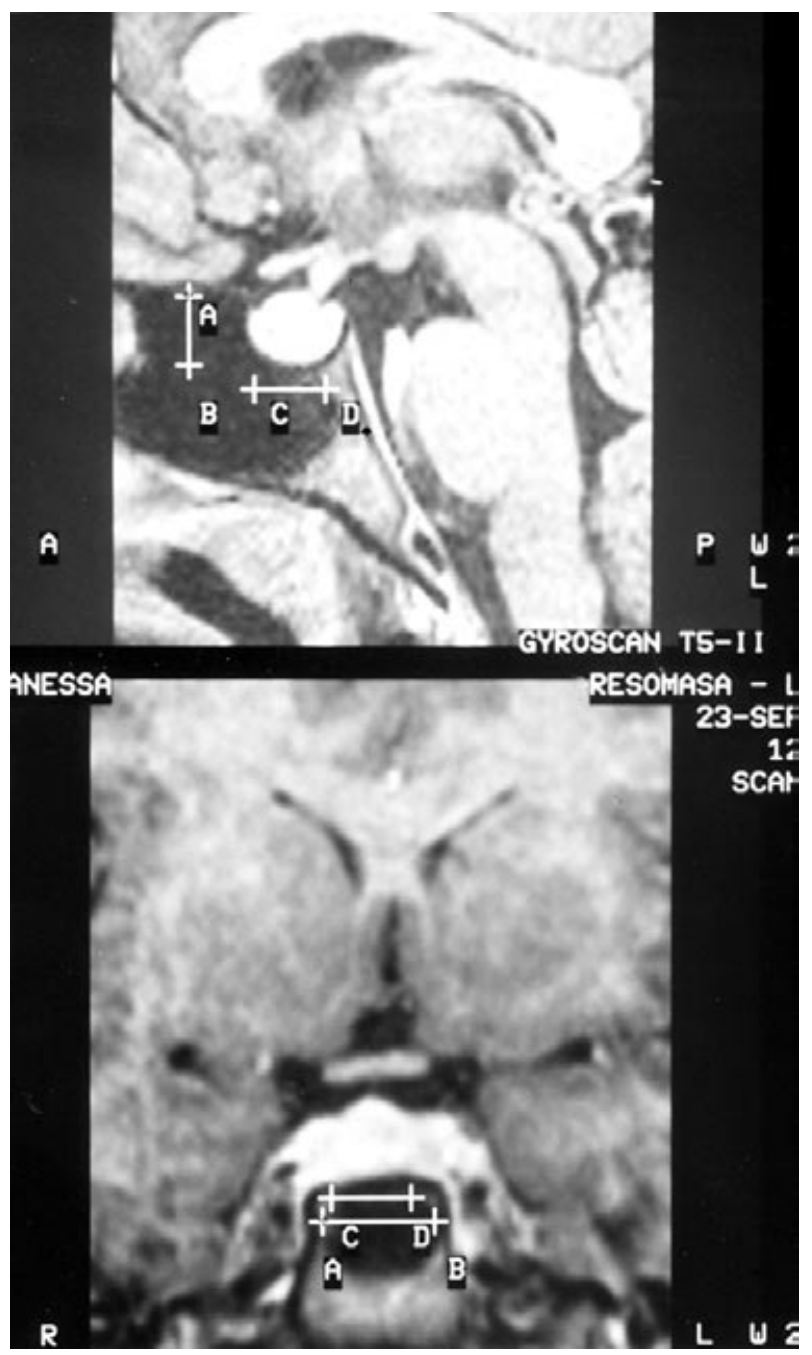

Figura 3. Resonancia magnética luego de 3 meses de iniciado el tratamiento con levotiroxina (100 ug/día).

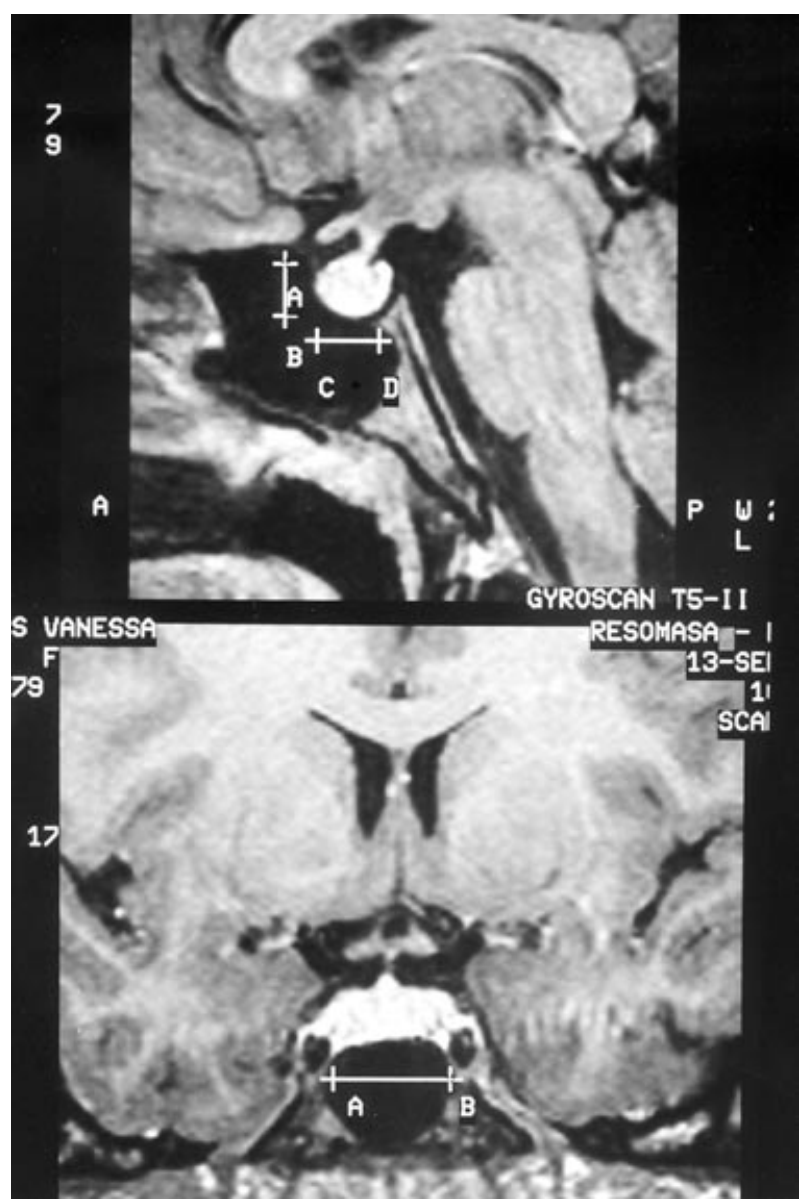

Figura 4. Resonancia magnética luego de dos años y cuatro meses de tratamiento.
- 20/05/95: RM cerebral: se informa como macroadenoma hipofisario de $12 \times 21 \times 12 \mathrm{~mm}$, que desplaza el quiasma óptico (figura 2); 10/06/95: campimetría: normal.

- 23/9/95: RM cerebral: formación tumoral selar en adenohipófisis de 9 $\mathrm{mm}$ de diámetro, luego de tres meses de tratamiento con levotiroxina en dosis de 100 ug diarios (figura 3).

- 16/11/95: Radiografia de manos: edad ósea de 17 años.

- 4/05/96 RM: microadenoma hipofisario $9 \mathrm{x} 8 \mathrm{~mm}$.
- 22/06/96: Prueba de perclorato a las 2 horas 32\%, a los 60 minutos $29,9 \%$, a los 120 minutos $13,2 \%$.

- 5/03/97: Anticuerpos antitiroideos antimicrosomales 1/40: anticuerpo antitiroglobulina negativo.

- 13/9/97: RM cerebral: hipofisis $7 \times 9 \times 17$ mm, 2 años y 4 meses postratamiento (figura 4).

Los valores hormonales de T4, T3, TSH y PRL (tabla 1) estuvieron dentro de límites normales.

\section{DISCUSIÓN}

Se presenta el caso de una adolescente que fue diagnosticada de hipotiroidismo primario e hiperprolactinemia secundaria al hipotiroidismo, quien recibió tratamiento irregular. Tuvo cefalea y pérdida de conocimiento, por lo que se decidió solicitar una resonancia magnética cerebral, en la que se encontró imagen sugestiva de 'macroadenoma hipofisiario'. En los casos de hiperplasia hipofisaria, el diagnóstico diferencial debe establecerse con prolactinoma o los raros adenomas productores de TSH ${ }^{(5,6)}$. Se ha descrito casos de cre- 
Tabla 1. Valores hormonales de PRL, TSH, T4 y T3 durante el periodo 1995 a 2011.

\begin{tabular}{|c|c|c|c|c|c|}
\hline Año & Fecha & PRL ng/ml & TSH uU/ml & T4 ug/dl & $\mathrm{T} 3 \mathrm{ng} / \mathrm{ml}$ \\
\hline \multirow[t]{7}{*}{1995} & 26.01 & 32 & & & \\
\hline & 25.03 & 18 & 4 & 9 & \\
\hline & 15.04 & 40 & 3,7 & 5 & \\
\hline & 15.05 & 28,6 & & & \\
\hline & 03.06 & & 27 & 4,6 & 110 \\
\hline & 22.08 & 29 & 0,4 & & \\
\hline & 11.11 & 8 & 0,15 & 8 & \\
\hline \multirow[t]{3}{*}{1996} & 24.01 & 11 & 1,4 & 9,5 & \\
\hline & 30.03 & & 1,9 & 10,2 & 110 \\
\hline & 13.07 & 13 & 3,4 & 7,5 & \\
\hline \multirow[t]{3}{*}{1997} & 05.01 & 12,5 & 64 & 4,2 & \\
\hline & 16.06 & & 0,32 & 12 & \\
\hline & 15.11 & & 0,3 & 11 & 135 \\
\hline 1998 & 21.09 & & 0,57 & 8,6 & \\
\hline \multirow[t]{2}{*}{2000} & 07.02 & & 0,54 & 10 & \\
\hline & 24.07 & 17 & 11 & 6,6 & \\
\hline 2003 & 02.01 & & 1,6 & 10 & \\
\hline \multirow[t]{2}{*}{2007} & 29.01 & 9 & 0,8 & 10,5 & \\
\hline & 17.05 & & 24,2 & 9,9 & 143 \\
\hline \multirow[t]{2}{*}{2011} & 08.08 & & 25 & 7,4 & 78 \\
\hline & 20.09 & & 1,8 & 9,7 & 123 \\
\hline
\end{tabular}

cimiento hipofisiario y pseudotumor debido a hipotiroidismo, tanto en población adulta como en pediátrica ${ }^{(7,8)}$.

Normalmente, las hormonas tiroideas actúan por medio de un mecanismo de retroalimentación negativa sobre la secreción hipotalámica de TRH; si la glándula tiroidea secreta una cantidad insuficiente de hormonas tiroideas, los niveles de TRH se incrementan. Esta elevación podría eventualmente resultar en hiperplasia de las células productoras de TSH y el crecimiento de la glándula pituitaria ${ }^{(5,9,10)}$.

La hiperplasia hipofisaria secundaria a hipotiroidismo puede manifestarse clínicamente de diversas formas: irregularidades menstruales, cefalea frontal de uno a dos meses de evolución, nocturna, progresiva, no asociada a vómitos, galactorrea o hipotiroidismo, generalmente asintomática ${ }^{(5,11)}$.
Los niños con hiperplasia e hipotiroidismo primario rara vez presentan síntomas neurológicos secundarios a la expansión selar $(3 \%){ }^{(5)}$. Después de largo tiempo de evolución, la hiperplasia hipofisaria puede producir lesiones irreversibles en la pituitaria, lo que puede llevar a deficiencia de una o más hormonas hipofisarias (hipopituitarismo) ${ }^{(5)}$.

En el hipotiroidismo primario, debido a la disminución de las hormonas tiroideas se incrementan los niveles de TRH, generando aumento de TSH; además, se produce hiperplasia de las células lactotrofas y como consecuencia hiperprolactinemia ${ }^{(9)}$; esto es debido al efecto estimulador de TRH sobre las células lactotrofas o por reducción del contenido de dopamina hipotalámica secundaria a la compresión del tallo hipofisario ${ }^{(12)}$. Generalmente, la clínica en estos pacientes es de hipotiroidismo severo y en algunos casos no es excesivamente sintomática. Los síntomas van a depender de la compresión mecánica local y son comúnmente deterioro del campo visual, hipertensión intracraneal, síntomas derivados de déficits hormonales, del tamaño de la porción tirotropa, que justifica el efecto compresivo mencionado; además, confiere a la hipófisis dimensiones variables, desde pocos milímetros hasta dimensiones de un macroadenoma, que a veces puede presentar extensión supraselar ${ }^{(9-11)}$.

Esta hiperplasia hipofisaria asociada a hipotiroidismo responde bien al tratamiento con levotiroxina, permitiendo no solo la resolución del hipotiroidismo, sino también de los signos clínicos asociados a esta ${ }^{(13)}$. La regresión de la tumoración hipofisaria puede producirse en corto plazo, aproximadamente luego de 4 semanas de iniciado el tratamiento ${ }^{(10,13)}$. En $81 \%$ de estos pacientes se ha encontrado hiperplasia de la glándula pituitaria, tanto en la TAC como en la RM, la cual se hace difícil de distinguir si es o no un adenoma hipofisario y, sin la interpretación endocrina adecuada, en muchos casos se ha realizado cirugías innecesarias ${ }^{(10,12)}$. En el caso de nuestra paciente, se evidenció inicialmente una imagen informada como 'macroadenoma hipofisario' de $12 \times 21 \times 12 \mathrm{~mm}$ (figura 2), con desplazamiento del quiasma óptico. Según la campimetría, no hubo compromiso de los campos visuales. Además, luego de recibir tratamiento con levotiroxina, se evidenció disminución del tamaño hipofisario, con mejoría clínica y bioquímica de la paciente, según controles tanto de resonancia magnética como de sus perfiles hormonales, desde el comienzo de su evaluación en 1995 hasta setiembre de 2011.

Las variaciones de las concentraciones de las hormonas tiroideas y TSH observadas durante todo el tiempo de seguimiento de esta paciente son debidas al abandono y reinicio temporal del tratamiento con levotiroxina (ver tabla 1). Desde agosto 2011, recibe una dosis de 175 ug/día. 
Se recomienda vigilar la evolución clínica desde el inicio del tratamiento para confirmar la regresión de la hiperplasia, la cual nos permite diferenciar entre hiperplasia y adenoma ${ }^{(12)}$. La reducción del tamaño hipofisario con ayuda de la RM nos demuestra la presencia de la hiperplasia hipofisaria, pero hay que tener en cuenta que el intervalo de tiempo necesario para la regresión de la hiperplasia en respuesta a la terapia de hormona tiroidea aún no ha sido definida ${ }^{(4)}$. Luego de tres meses de iniciado el tratamiento con levotiroxina, se observó una disminución del volumen hipofisario (figura 3). Otro aspecto a tomar en cuenta en la evolución clínica es la respuesta a la terapia hormonal, la cual debe ser estrechamente vigilada ante la rara posibilidad de presentar manifestaciones neurológicas agudas, tales como desarrollo de pseudotumor cerebral y falla visual paradojal inducida por la terapia hormonal tiroidea, o la posibilidad de un tumor hipofisario no tirotropo que no regresiona con el tratamiento adecuado ${ }^{(4)}$.

Nuestra paciente tiene un diagnóstico de hipotiroidismo primario, sin ninguna etiología precisada. La causa de hiperplasia tirotropa más frecuentemente detectada, tanto en niños como en adultos, ha sido la tiroiditis crónica autoinmune, y congénita (agenesia/ hipoplasia tiroidea o dishormonogénesis) ${ }^{(11)}$. En la paciente se determinó los títulos de anticuerpos antitiroideos, los cuales no mostraron valores muy elevados. Cuando la captación de I-13I es normal o alta, como en el caso de nuestra paciente, debe procederse a la realización de una prueba de descarga con perclorato ${ }^{(14)}$, que es una prueba diagnóstica que evalúa la capacidad del perclorato para reducir la permanencia de iodo radiactivo en la tiroides. Para hacer la prueba, se administra yodo marcado y 2 horas después se indica perclorato $(10 \mathrm{mg} / \mathrm{kg}$ de perclorato potásico vía oral), para posteriormente medir la cantidad de yodo marcado en la tiroides. El perclorato bloquea o inhibe rápidamente el atrapamiento del yoduro, con el resultado de que la captación del yodo radiactivo alcanza un valor meseta a los 5 a 10 minutos. Normalmente, el yodo intracelular se oxida y se organifica rápidamente; sin embargo, si existe un defecto de organificación, el yodo intracelular no organificado (inorgánico) se escapa de la glándula, es decir, se produce una descarga de perclorato. La prueba es considerada positiva cuando se produce una descarga mayor de $20 \%$ del yodo radiactivo total de la glándula medida luego de la administración del perclorato ${ }^{(14)}$. En nuestra paciente, la prueba de perclorato fue negativa, lo cual descartó un defecto de organificación.

La cirugía debe reservarse para la descompresión del quiasma óptico o para obtener un diagnóstico patológico, en el caso de una tumoración de la pituitaria que no responde o empeora a pesar del reemplazo con hormona tiroidea. En tal caso, debería reevaluarse la posibilidad diagnóstica.

\section{REFERENCIAS BIBLIOGRÁFICAS}

1. Castillo O, Tello L. Hiperplasia hipofisaria tratada con levotiroxina. Tema presentado en el XIV Congreso Panamericano de Endocrinologia; Cancún, México, 21 a 25 de noviembre de 1997. Resumen No 12.

2. Lee CY, Hsu HH, Lai HY, Lee ST. Rapid progression of hypothyroidism-related pituitary hyperplasia case report. J Neurosurg Pediatr. 2008;2(3):212-4.

3. Eom KS, See-Sung C, Kim JD, Kim JM, Kim TY. Primary hypothyroidism mimicking a pituitary macroadenoma: regression after thyroid hormone replacement therapy. Pediatr Radiol. 2009;39(2):164-7.

4. Sarlis NJ, Brucker-Davis F, Doppman JL, Skarulis MC. MRI-demonstrable regression of a pituitary mass in a case of primary hypothyroidism after a week of acute thyroid hormone therapy. J Clin Endocrinol Metab. 1997;82(3):808-11.

5. Franceschi R, Rozzanigo U, Failo R, Bellizzi M, Di Palma A. Pituitary hyperplasia secondary to acquired hypothyroidism: case report. Ital J Pediatr. 2011;37:15-8.

6. Horvath E, Kovacs K, Scheithauer BW. Pituitary hyperplasia. Pituitary. 1999;1(3-4):169-80.

7. Young M, Kattner K, Gupta K. Pituitary hyperplasia resulting from primary hypothyroidism mimicking macroadenomas. Br J Neurosurg. 2009;13(2):13842.

8. Kocova M, Netkov S, Sukarova-Angelovska E. Pituitary pseudotumor with unusual presentation reversed shortly after the introduction of thyroxine replacement therapy. J Pediatr Endocrinol Metab. 2001;14(9):1665-9.

9. Erem C. A case of pituitary hyperplasia secondary to primary hypothyroidism presenting with irregular menses, galactorrhea, and hyperprolactinemia. Endocrinologist. 2010;20(4):168-70.

10. Passeri E, Tufano A, Locatelli M, Lania AG, Ambrosi $\mathrm{B}$, Corbetta S. Large pituitary hyperplasia in severe primary hypothyroidism. J Clin Endocrinol Metab. 2011;96(1):22-3.

11. Guerrero-Fernandez J, Benzanilla C, Orio M, Carceller F, Heredero JJ, Gracia R. Tumoración hipofisiaria secundaria a hipotiroidismo primario: un caso de hiperplasia tirotropa que estimula un adenoma hipofisiario. An Pediatr( Barc). 2008;69(2):189-90.

12. Simsek E, Simsek T, Savas-Erdeve S, Erdogmus B, Dôsoglu M. Pituitary hyperplasia mimicking pituitary macroadenoma in two adolescent patients with long-standing primary hypothyroidism: case reports and review of literature. Turk J Pediatr. 2009;51:62430.

13. García J, Polanía DL, Builes CA. Hiperplasia hipofisiaria secundaria a hipotiroidismo primario. Acta Med Colomb. 2005;30(2):65-7.

14. Mayayo E, Ferrández Longás A, Labarta JI. Interpretación de las pruebas tiroideas. An Esp Pediatr. 2002;56[Supl 4]:42-52.

Trabajo presentado en parte y publicado como resumen en el XIV Congreso Panamericano de Endocrinología, Cancún, México, noviembre 1997. Artículo recibido el 14 de agosto de 2012 y aceptado para publicación el 14 de octubre de 2012.

\section{Correspondencia:}

Oscar Alejandro Castillo Sayán

Dirección: Instituto Nacional de Biología Andina, UNMSM

Av. Alfonso Ugarte 848, Lima 1.

Telefax: 4313355.

Correo electrónico: oscar.oculm@gmail.com 